\title{
UM FILTRO GAUSSIANO ADAPTATIVO PARA ANÁLISE COMPARATIVA DE IMAGENS DE RADARES E SATÉLITES METEOROLÓGICOS
}

\author{
João Victor Cal Garcia ${ }^{1}$; Stephan Stephany ${ }^{1}$; Roberto Vicente Calheiros ${ }^{2}$; Augusto \\ Brandão d'Oliveira ${ }^{3}$ \\ ${ }^{1}$ Laboratório Associado de Computação e Matemática Aplicada (LAC) Instituto Nacional de Pesquisas Espaciais \\ (INPE) - São José dos Campos \\ ${ }^{2}$ Instituto de Pesquisas Meteorológicas - IPMet/UNESP - Bauru \\ ${ }^{3}$ Departamento de Ciências de Computação e Estatística- UNESP - São José do Rio Preto
}

\section{RESUMO}

A aplicação do radar meteorológico em irrigação vem tendo crescente desenvolvimento. $\mathrm{O}$ uso sinérgico de informações de outros sensores destacando-se ai as de satélites meteorológicos, agrega valor significativo em muitos casos. A análise conjunta de imagens de satélites e radares meteorológicos permite correlacionar estruturas precipitantes observadas em ambas as imagens. Essa análise tornaria viável a extensão do alcance de radares em meteorológicos em terra. Entretanto, além das resoluções espaciais e temporais diferentes dessas imagens, análise comparativa é dificultada devido a efeitos de rotação, distorção geométrica e aos diferentes formatos de dados, projeções geográficas e sistemas de coordenadas. Neste trabalho, emprega-se uma abordagem baseada num filtro gaussiano adaptativo para comparação dessas imagens. Os resultados estatísticos obtidos da comparação são confrontados com aqueles obtidos por outros métodos existentes.

UNITERMOS: irrigação, filtragem de imagens, radar meteorológico, satélite meteorológico

\section{GARCIA,J.V.C. ${ }^{1}$; STEPHANY,S. ${ }^{1}$; CALHEIROS,R.V. ${ }^{2}$; D'OLIVEIRA,A.B. ${ }^{3}$ AN ADAPTIVE GAUSSIAN FILTER FOR THE COMPARATIVE ANALYSIS OF IMAGES FROM METEOROLOGICAL RADAR AND SATELLITES.}

\section{ABSTRACT}

Meteorological satellite and radar data comparative analysis allows to correlate the precipitation structures observed in both images. Such analysis would make feasible the extension of the range of ground-based meteorological radars. In addition to the different spatial and temporal resolution of these images this comparative analysis presents difficulties due to the effects of rotation and distortion, besides the different formats, projections, and coordinate systems. This work employed an approach based on a Gaussian adaptive filter in order to compare such images. The statistical results obtained from the comparison of the images are matched to those produced by other methods. 
KEYWORDS: irrigation, image filtering, meteorological radar, meteorological satellite.

\section{INTRODUÇÃO}

O conhecimento mais detalhado do campo de precipitação é de grande relevância para as práticas de irrigação. Ferramenta básica para tal conhecimento o radar meteorológico, de per si ou em combinação com outros sensores, tem relevante aplicação na irrigação.

No exterior, por exemplo, imagens de chuva acumulada derivadas das observações dos radares de Shannon e Dublin - fornecidas pelo Serviço Meteorológico da Irlanda - têm sido cada vez mais utilizadas no gerenciamento de irrigação (http://www.met.ie/climate/9data 07.asp).

E, emblematicamente, a patente n.6850819 emitida em 01 de fevereiro de 2005 nos E.U.A. sobre um sistema de controle de irrigação envolve o exame de "dados de chuva de uma varredura de radar" de pelo menos de uma primeira área de acordo com critérios predeterminados..." (http://www.potentstorm.us/patents/6850819/claims.html).

No Brasil, já nas atividades relativas ao Projeto RADASP - Meteorologia com Radar em São Paulo (1982), inclina-se a prestação de informação específica de radar (chuva) para as áreas com pivôs de irrigação (inicialmente) para a região de Ilha Solteira.

O estudo das imagens de um mesmo evento de precipitação obtidas por instrumentos diferentes muitas vezes é dificultado pela forma como essas imagens são apresentadas. Para que seja possível fazer um estudo comparativo pixel a pixel, é necessário corrigir os efeitos de rotação e da diferença de resolução já esperados, além das distorções geométricas particulares para cada imagem.

Muitos avanços já foram realizados nesse campo. Existem diversos métodos para a rotação de imagens, bem como para a mudança de resolução, mas a maioria deles não leva em consideração os efeitos devidos à distorção geométrica (Gonzalez \& Woods, 2007 e Jain, 1989).

Em um estudo específico a respeito da correlação de imagens de satélite e radar meteorológico, o trabalho de Calheiros \& d'Oliveira (2007) apresentou uma maneira de lidar com o problema levando em conta a distorção geométrica da imagem de menor resolução. No caso estudado, as imagens de satélite possuíam uma resolução mais de vinte vezes menor em cada dimensão em relação àquelas de radar. Além disso, a distorção presente nas imagens de radar poderia ser desconsiderada ao se comparar com a distorção existente nas imagens de satélite.

O presente trabalho é uma generalização da técnica proposta em Calheiros \& d'Oliveira (2007) para outros tipos de imagem, levando em consideração os efeitos de distorção presentes em ambas as imagens.

\section{MATERIAIS E MÉTODOS}

A fim de facilitar a leitura, a imagem de maior resolução será referida doravante como Imagem Fonte, e a imagem pós-processada será referida como Imagem Objetivo, e a imagem de menor resolução será referida como Imagem Modelo. Além disso, cada pixel das Imagens Fonte, Modelo e Objetivo serão referidos respectivamente como $F(x, y), M(x, y)$ e $O(x, y)$. 
O procedimento descrito no presente trabalho visa a redução da resolução da Imagem Fonte para a mesma resolução da Imagem Modelo, criando assim a Imagem Objetivo. Além disso, a área no mundo real representada por cada pixel da Imagem Objetivo deve ser o mais próxima possível da área representada pelo pixel equivalente da Imagem Modelo, ou seja, deverão ter distorções geométricas semelhantes.

Ao se reduzir a resolução de uma imagem utilizando métodos como vizinho mais próximo ou o bi-cúbico, os pixels são considerados entidades pontuais, sem dimensão real. Como resultado, a Imagem Objetivo gerada por esses processos desconsideraria a distorção geométrica de ambas as Imagem Fonte e Imagem Modelo. Um eventual estudo comparativo pixel a pixel entre as imagens Objetivo e Modelo apontaria um grau maior de disparidade, já que pixels equivalentes estariam representando áreas diferentes no mundo real (Jain, 1989 e Jensen \& Lulla, $1987)$.

\subsection{Filtro gaussiano elíptico não-linearmente variável}

A fim de re-mapear a Imagem Fonte de maneira que sua distorção geométrica e resolução sejam equivalentes à da Imagem Modelo, o trabalho de Calheiros \& d'Oliveira (2007) propõe o uso de um filtro para suavizar desigualmente a imagem antes de reduzir sua resolução.

$$
\text { Filtro }_{i, j}(x, y)=\frac{\left.\exp \left(-\left(\frac{x-\frac{a_{i}}{2}}{2}\right)^{2}+\left(\frac{y-\frac{b_{j}}{2}}{2}\right)^{2}\right)\right)}{\sum_{w \rightarrow 0}^{a_{1}} \sum_{z=0}^{b_{j}} F_{i l \text { tro }}(w, z)}
$$

O valor assumido por cada pixel em uma imagem possui dependência em relação ao valor de seus pixels vizinhos Gonzalez \& Woods (2007). Simplificadamente pode-se assumir que o valor de cada pixel influencia o valor de seus vizinhos na forma de uma distribuição gaussiana. Calheiros \& d'Oliveira (2007) utilizam dessa característica propondo um filtro gaussiano que varie pixel a pixel na Imagem Fonte, influenciando desigualmente o valor de cada pixel em relação ao valor de seus vizinhos.

O objetivo desse filtro é suavizar a Imagem Fonte de forma a introduzir uma nova distorção geométrica equivalente à da Imagem Modelo. Para tanto, o filtro é composto utilizando a Equação 1, onde $(x, y)$ representam o $x, y$-ésimo pixel da Imagem Fonte, $\mathrm{a}_{\mathrm{x}}$ é o tamanho do filtro no eixo $x$ enquanto $\mathrm{a}_{\mathrm{y}}$ é o tamanho do pixel no eixo $y$. Tanto $\mathrm{a}_{\mathrm{x}}$ quanto $\mathrm{a}_{\mathrm{y}}$ são calculados de forma que o filtro tenha, ao sobrepor-se na Imagem Fonte, o tamanho aproximado que um pixel da Imagem Modelo possui na região equivalente, conforme é mostrado na a Figura 1. 


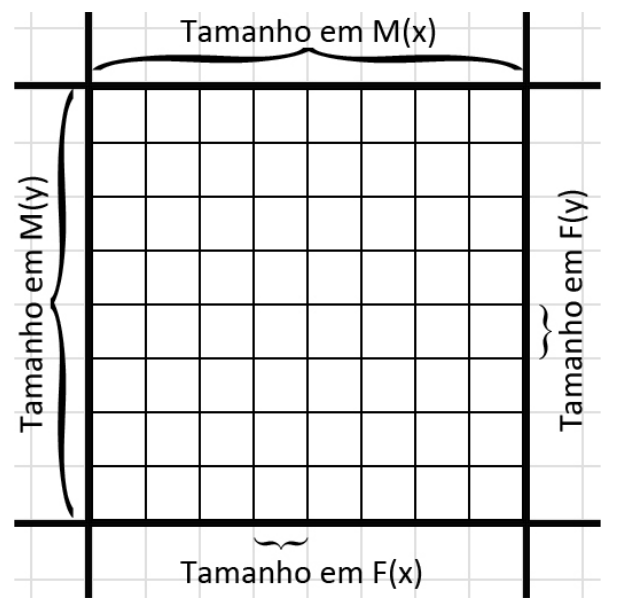

Figura 1. Comparação do tamanho dos pixels

Ao se repetir esse processo em toda a Imagem Fonte, a imagem resultante não só está suavizada de maneira a possuir resolução virtualmente equivalente à da Imagem Modelo, mas também suas distorções geométricas são semelhantes.

\subsection{Re-mapeamento}

A partir desse ponto, o re-mapeamento é feito utilizando o método do vizinho mais próximo. Para cada pixel da Imagem Fonte, associa-se o pixel da Imagem Modelo cujas coordenadas no mundo real do centro estejam mais próximas de seu próprio centro, conforme a Figura 2. Dessa forma, cada pixel $i$ da Imagem Modelo será associado à pixels da Imagem Fonte.

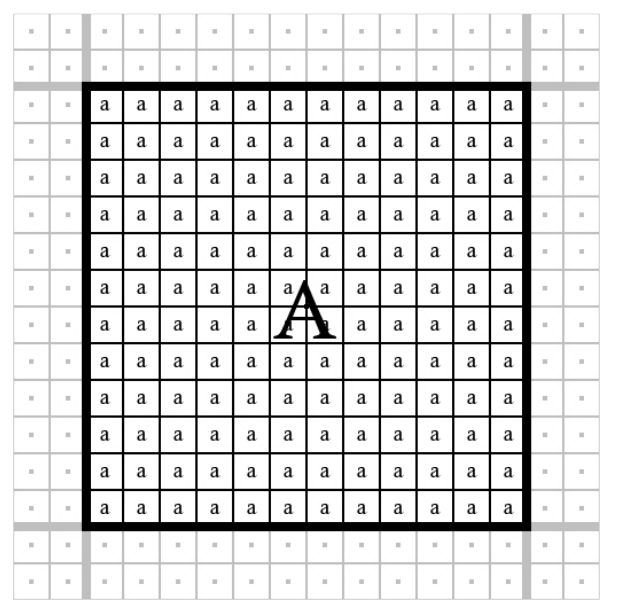

Figura 2. Associação dos Pixels da Imagem Modelo com os da Imagem Fonte

Como a Imagem Modelo e a Imagem Objetivo possuem a mesma resolução, basta então calcular a média do valor dos pixels e atribuir esse valor ao pixel equivalente $i$ na Imagem 
Modelo. Assim, a imagem resultante possui a mesma resolução que a Imagem Modelo, além de seus pixels estarem numa mesma distorção geométrica.

\section{RESULTADOS E DISCUSSÃO}

A fim de validar o método apresentado, é necessário comparar não somente a Imagem Modelo com a Imagem Objetivo, mas também comparar outras técnicas. Baseado no trabalho de Mecklenburg et al. (2000), foram utilizadas os parâmetros $P_{1}, P_{4}, P_{5}$ e $P_{6}$ para uma comparação inicial. O parâmetro $P_{1}$ é obtido calculando a diferença do centro de massa das duas imagens, $P_{4}$ é a razão entre as medianas das imagens, $P_{5}$ é dado pela razão entre a diferença (25quantil - 75-quantil) de cada imagem, enquanto $P_{6}$ é calculado através da correlação de ambas as imagens. As imagens comparadas são consideradas mais semelhantes conforme o valor de $P_{1}$ tender a zero, $P_{4}, P_{5}$ tenderem à $100 \%$ e o valor de $P_{6}$ tender a um (Mecklenburg et al, 2000).

Foram utilizados dois conjuntos de imagens para esse trabalho. O primeiro conjunto é composto de oito imagens geradas por radares meteorológicos banda $\mathrm{S}$ em terra como Imagem Fonte, e as oito imagens do canal 5 do instrumento $M H S$ do satélite NOAA-18 dos mesmos eventos como Imagem Modelo. No segundo conjunto, foram utilizadas imagens de composições 3D geradas por computador, sendo que o conjunto Imagem Modelo possui resolução significativamente menor e a cena foi levemente distorcida em cada caso. A imagem original equivale à Figura 3.

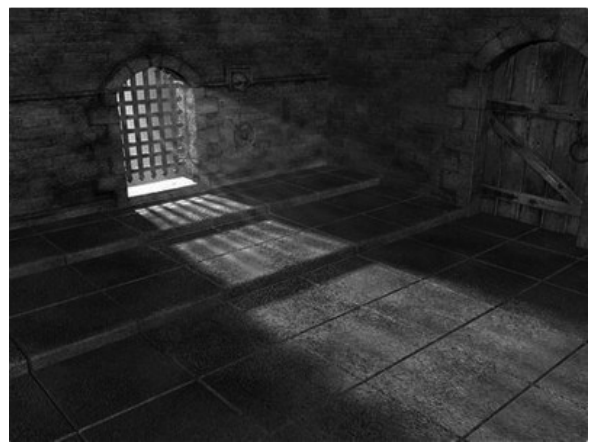

Figura 3. Composição 3D sem distorção e em resolução máxima

Os métodos utilizados para a comparação foram o proposto pelo presente trabalho, Redução de resolução utilizando o re-mapeamento ponderado proposto sem filtragem, redução de resolução do vizinho mais próximo, redução de resolução bi-cúbica e redução de resolução bilinear. Os resultados para uma das imagens de composição 3D podem ser visualizados na Figura 4, apenas como exemplo. 

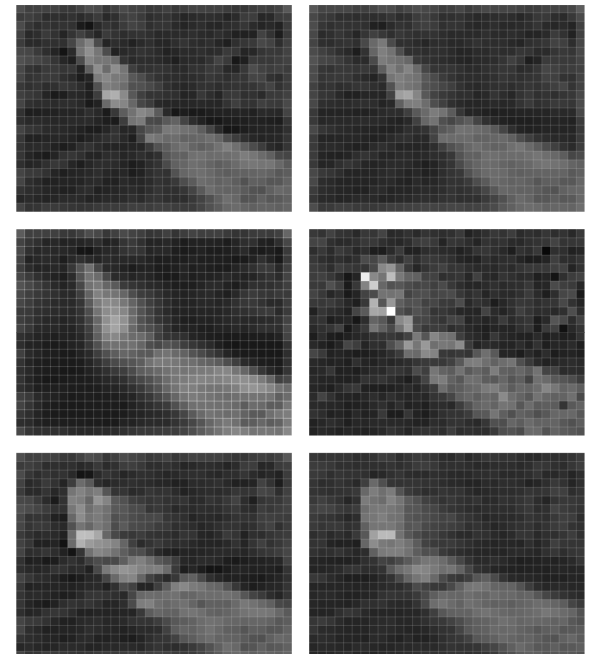

Figura 4. De cima para baixo, da esquerda para a direita: Imagem Modelo original; Calheiros \& d’Oliveira; Re-Mapeamento; Vizinho mais Próximo; Bi-cúbica; Bi-linear

De acordo com a Tabela 1, a técnica proposta por Calheiros \& d'Oliveira (2007) obteve os melhores resultados nos quatro parâmetros propostos por Mecklenburg et al (2000). Isso demonstra que a distorção geométrica das imagens pode influenciar fortemente no processo de redução de resolução.

Tabela 1. Resultados comparativos

\begin{tabular}{ccccc}
\hline Método utilizado & $\mathrm{P}_{1}$ & $\mathrm{P}_{4}$ & $\mathrm{P}_{5}$ & $\mathrm{P}_{6}$ \\
\hline Calheiros \& d'Oliveira & 0.013248 & 87.18125 & 91.10800 & 0.97420 \\
Re-mapeamento ponderado & 0.186221 & 42.41018 & 93.11111 & 0.75539 \\
Vizinho mais próximo & 2.323841 & 22.54060 & 45.02028 & 0.56262 \\
Bi-cúbico & 0.782121 & 74.41018 & 84.12097 & 0.81096 \\
Bi-linear & 1.842132 & 51.42065 & 48.70101 & 0.74017 \\
\hline
\end{tabular}

O método bi-cúbico obteve um resultado bem interessante, seguido pelo método bilinear, mas a distorção geométrica ainda teve grande influencia em ambos os casos. No geral, no entanto, esses dois métodos são mais rápidos que o de Calheiros \& d'Oliveira (2007), e deveriam ser considerados em situações onde a precisão é menos importante que o tempo de processamento.

Usando apenas o remapeamento conforme proposto na mesma técnica, ainda se obteve um resultado bastante significativo, principalmente se comparado ao desempenho do método do vizinho mais próximo. É importante notar que essas duas técnicas mais simples são bastante semelhantes, além de serem computacionalmente menos custosas que as outras utilizadas.

\section{1 aplicação a meteorologia com satélites}

Na meteorologia, radares e satélites podem ser utilizados no estudo de precipitações. Por serem instrumentos de naturezas distintas que observam o mesmo evento de maneiras diferentes, 
é esperado que seus dados não sejam diretamente equivalentes.

No trabalho de Calheiros \& d'Oliveira (2007), é apresentado um estudo das relações entre as imagens geradas por radar e por satélite de um mesmo evento de precipitação.

$\mathrm{O}$ radar estudado opera em banda $\mathrm{S}$, situado nas proximidades da cidade de Bauru/SP, latitude $22^{\circ} 21^{\prime} 3 " \mathrm{~S}$ e longitude $49^{\circ} 1^{\prime} 42^{\prime \prime} \mathrm{W}$, e gerava imagens CAPPI de $480 \times 480$ pixels. Cada pixel equivale à aproximadamente $1 \mathrm{~km}^{2}$ no mundo real e apresenta dados em decibéis de $\mathrm{Z}$.

O satélite utilizado foi o NOAA-18, de órbita polar. Os dados estudados são do canal 5 do instrumento MHS, obtidos em imagens com pixels cujo tamanho varia entre $10 \mathrm{~km}$ e $35 \mathrm{~km}$ apresentando temperatura de topo de nuvem. Em média, uma sub-matriz de $26 \times 26$ pixels equivale à mesma área no mundo real da imagem de radar. A Figura 5 apresenta as imagens de radar e satélite.

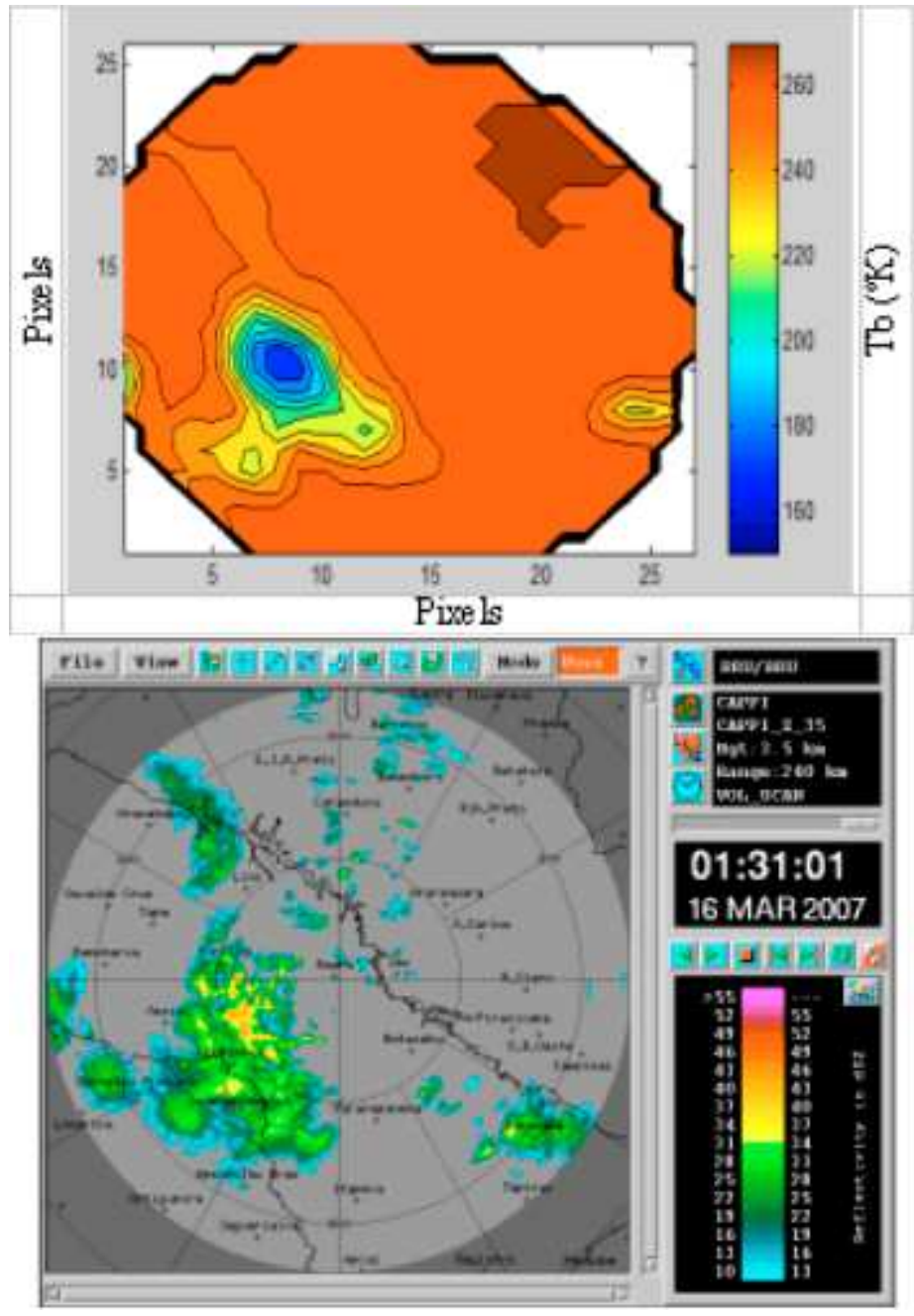

Figura 5. Em cima imagem do Canal 5 do MHS, NOAA18, Temperatura de brilho. Em baixo : imagem do mesmo evento visto pelo radar de Bauru.O evento mostrado é do dia 16/03/2007, 01h31min GMT 
Devido à grande diferença entre a resolução de cada imagem e da variação nas imagens de satélite área representada por cada pixel, a técnica proposta em Calheiros \& d'Oliveira (2007) para a filtragem de imagens se mostra apropriada. Na Figura 6 é dado um exemplo de como a aplicação do processo de filtragem permite auxiliar na comparação direta (pixel a pixel) de duas imagens de instrumentos diferentes, bastando conhecer a área que cada pixel representa no mundo real.

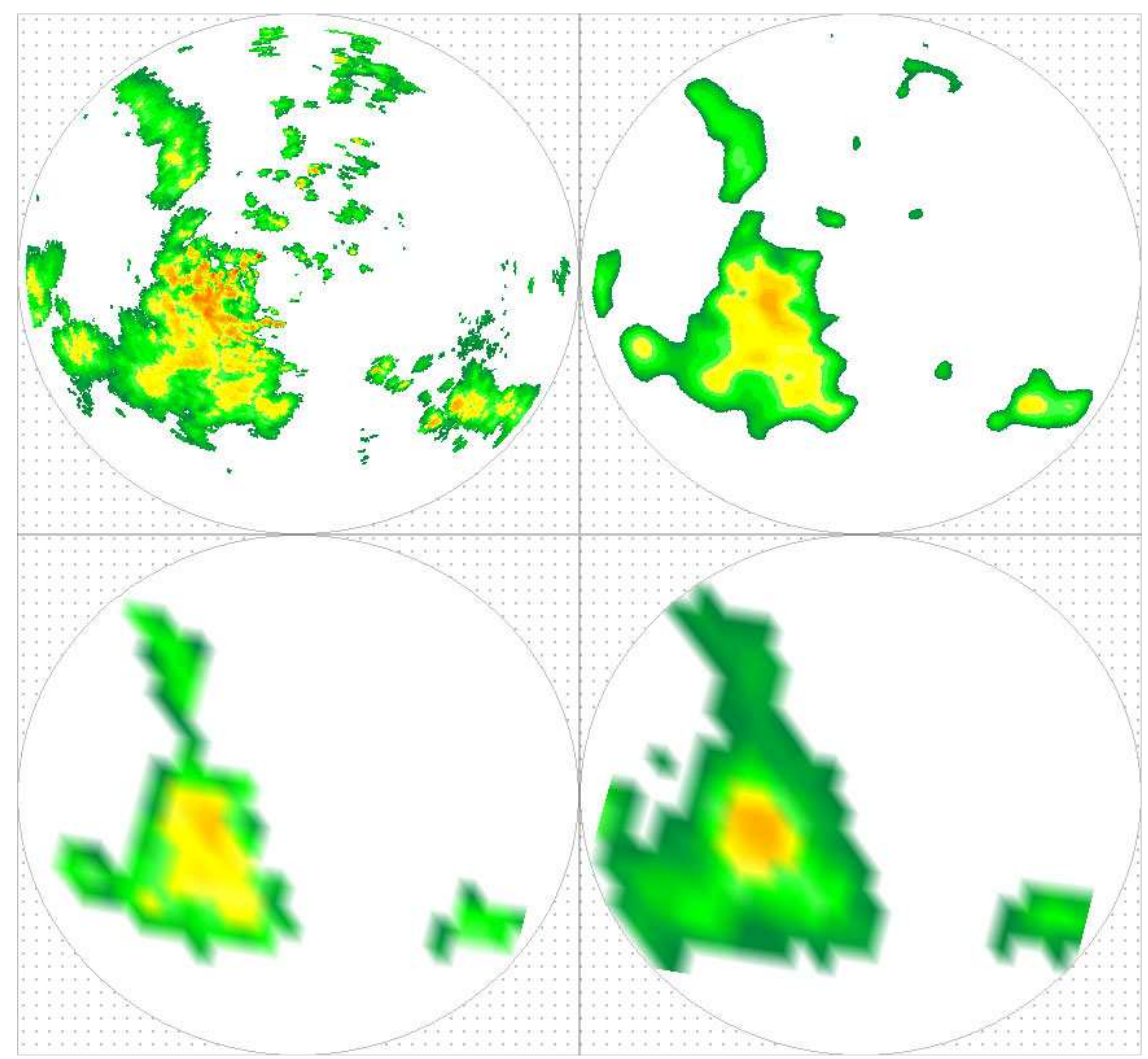

Figura 6. Em cima à esquerda: Imagem de Radar não filtrado, em cima à direta, radar somente filtrado, em baixo à esquerda radar filtrado e com resolução reduzida e em baixo à direita: imagem de satélite. O círculo mostrado tem raio de $240 \mathrm{~km}$ e seu centro corresponde à cidade de Bauru, onde está localizado o radar. O evento mostrado é do dia 16/03/2007, 01h31min GMT

Uma relação $\mathrm{Tb}-\mathrm{Z}$ é aplicada aos dados de satélite para que eles apresentem o mesmo tipo de unidade que os de radar (Calheiros \& d'Oliveira 2007). A Tabela 2 apresenta os valores dos parâmetros estatísticos de Mecklenburg et al. (2000) obtidos ao aplicar o processo de filtragem e ajuste de resolução no evento apresentado na Figura 5.

O parâmetro $P_{1}$ passa, nesse caso, a ser apresentado em quilômetros, mostrando que a distância entre o centro de massa da imagem de radar filtrada e de satélite ajustada é de pouco mais de 25 metros. Os parâmetros $P_{4}$ e $P_{5}$ apresentam valores altos, indicando que as variações 
de intensidades são semelhantes nas duas imagens. Deve-se notar que o valor de $P_{5}$ não ser considerado melhor que $100 \%$ - ele é equivalente a 75.777025, mas o valor foi mantido para preservar a informação que a diferença (25-quantil - 75-quantil) é menor na imagem de radar que de satélite. $\mathrm{O}$ parâmetro $P_{6}$ também possui um valor alto, o que indica uma grande correlação. Analisando todas essas informações, é de se concluir que a aplicação conjunta da relação Tb-Z e do procedimento de filtragem gera duas imagens bem parecidas.

\begin{tabular}{cc}
\multicolumn{2}{c}{ Tabela 2. Valores comparativos } \\
\hline Variável & Valor Obtido \\
\hline $\mathrm{P}_{1}$ & 0.026337 \\
$\mathrm{P}_{2}$ & 89.452300 \\
$\mathrm{P}_{3}$ & 131.966120 \\
$\mathrm{P}_{4}$ & 0.817160 \\
\hline
\end{tabular}

\section{CONCLUSÕES}

O método proposto por Calheiros \& d'Oliveira (2007) possui um desempenho bem mais interessante no que se diz respeito a reduzir a resolução de imagens levando em conta a distorção geométrica da imagem alvo, quando é necessário comparar duas imagens diferentes pixel a pixel.

Computacionalmente, esse método é mais custoso que todos os outros utilizados na comparação, apesar de ainda serem necessários estudos que quantifiquem esse fator.

Também seria interessante averiguar em trabalhos futuros a qualidade do método para reduzir a resolução de imagens sem distorção geométrica, o que poderia auxiliar à qualificação do mesmo para situações genéricas. Com a incorporação da recuperação da estrutura das células de radar a grande distância às práticas operacionais do IPMet, será significativamente ampliada a área de uso potencial dos radares de Bauru e Presidente Prudente em irrigação.

\section{REFERÊNCIAS}

CALHEIROS, R. V. Meteorologia com Radar em São Paulo: Projeto RADASP/FAPESP.1982.

CALHEIROS, R. V. ; D'OLIVEIRA, A. B. Radar-satellite retrieval of cell structure: impact of Tb-Z relationships. Geophysical Research Abstracts, volume 9, page 10621. 2007.

GONZALEZ, R. C. ; WOODS, R.E. Digital image fundamentals. 1987.

JAIN, A. Fundamentals of digital image processing. Prentice-Hall, Inc. Upper Saddle River, NJ, USA. 1989.

JENSEN, J. ; LULLA, K. Introductory digital image processing: a remote sensing perspective. Geocarto International, v. 2, n. 1, p.65. 1987. 
MECKLENBURG, S. ; JOSS, J., ; SCHMID, W. Improving the nowcasting of precipitation in an Alpine region with an enhanced radar echo tracking algorithm. Journal of Hydrology, v. 239, n. $1 / 4$, p.46-68. 2000. 